\title{
THE MIN CONE OVER THE CIRCLE GROUP
}

\author{
J. E. L'HEUREUX
}

Abstract. It is shown that the min cone over the circle group is the only inverse semigroup or Clifford semigroup on the 2-cell with an identity whose set of idempotents has a cut point.

A topological inverse semigroup $S$ is a Hausdorff space together with a continuous associative multiplication in which each element has a unique inverse and whose idempotents commute. Such semigroups were studied in [5], which includes a characterization of inverse semigroups whose underlying space is an arc.

Considering inverse semigroups on the 2-cell, the purpose of this paper is to show that the min cone over the circle group is the only inverse semigroup with an identity on the 2-cell whose set of idempotents has a cut point. Unless otherwise stated, $S$ will denote such a semigroup and $E$ its set of idempotents. The closure of $A$ will be denoted by $A^{*}$ while other notations, definitions, and preliminary results can be found in [2], [3], and [5].

The existence of the kernel $K$ in compact semigroups is well known, as are the facts that $K$ is connected if $S$ is connected, and that $K$ consists of one $\mathscr{D}$-class. It follows that $K$ is a single point and hence $S$ has a zero.

LeMMA 1 . If $e$ is a cut point of $E$, then $H(e)$ is a circle group.

Proof. Let $r$ be the retraction of $S$ onto $E$ defined by $r(s)=s s^{-1}$ [5]. Thus $r^{-1}(e)=R(e)$, the $\mathscr{R}$-class of $e$, separates $S$ and it follows that $\operatorname{dim} R(e)=\operatorname{dim} D(e)=1$ [3], [4]. Inversion restricted to $R(e)$ is a homeomorphism onto $L(e)$, so $\operatorname{dim}(L(e) \times R(e))=2$. The multiplication map $m$ from $L(e) \times R(e)$ onto $D(e)$ lowers dimension by one, so it follows that there are elements $t \in L(e)$ and $s \in R(e)$ such that $m^{-1}(t s) \subset H(t) \times H(s)$ and $\operatorname{dim} m^{-1}(t s) \geqq 1$; hence $\operatorname{dim} H(t)=\operatorname{dim} H(s)=1$. By Green's translational lemmas, $H(e)$ is homeomorphic to $H(s)$, hence $\operatorname{dim} H(e)=1$ and $H(e)$ is a circle group [1].

Let $T$ be a min thread from 1 to 0 [6]; clearly $T$ contains $e$. By Exercise 7, p. 209 of [3], $S e S=e S e$ is a min cone over the circle group. In fact there

Received by the editors February 16, 1973.

AMS (MOS) subject classifications (1970). Primary 22A15; Secondary 22A99.

Key words and phrases. Topological inverse semigroups, 2-cell.

(c) American Mathematical Society 1973 
is a maximal idempotent of $T$, say $f$, such that $S f S=f S f$ is a min cone over the circle group. Let $e$ now denote this maximal idempotent. If $H(e)=\mathrm{Bd} S$ then $e=1$ and we are through. Therefore suppose $e \neq 1$ and let $T_{1}=\{t \in T: e \leqq t\}$ and $C=\{s \in S: s e=e\}$. Under this assumption, $E \backslash S e S \neq \varnothing$ and has no cut points, so let $e_{0}$ denote an interior point of $E \backslash \mathrm{SeS}$.

Lemma 2. The center of $S$ contains $S e S$.

Proof. For $x \in S \mid S e S$ and $f \in E \cap S e S, f \leqq x x^{-1}$ and $x f x^{-1} \in E \cap H(f)$; but $H(f)$ is a group, so $x f x^{-1}=f$ and $x f=f x$. If $t \in S e S$, then $t x=$ $\left(t t^{-1} t\right) x=t\left(t^{-1} t\right) x=\left(t^{-1} t x\right) t$ since $t^{-1} t x \in H(t)$ and $S e S$ is commutative. Now $t^{-1} t x=x t^{-1} t$ since $t^{-1} t=t t^{-1} \in E \cap S e S$, so $t x=\left(t^{-1} t x\right) t=\left(x t t^{-1}\right) t=x t$.

LEMma 3. The set $C$ is a closed connected acyclic inverse subsemigroup of $S$.

Proof. For $c \in C, c T_{1} \subset C$ and is an arc from $c$ to $e$. Therefore $C$ is connected and it is easily shown that $C$ is a closed connected inverse subsemigroup. The mapping $H$ from $T_{1} \times C$ onto $C$ defined by $H(t, c)=t c$ is continuous, where $H(1, c)=c$ and $H(e, c)=e$ for all $c \in C$. The generalized homotopy theorem [7] implies $H^{n}(C) \approx H^{n}(e)$ for all $n$, hence $C$ is acyclic.

Lemma 4. $1 \notin(S \backslash C)^{*}$.

Proof. If $\left\{t_{n}\right\}$ is a sequence in $S \backslash C$ converging to 1 , then $\left\{t_{n} e_{0}\right\}$ converges to $e_{0}$ and there exists an $m$ such that $t_{m} e_{0} \in E \backslash S e S$. Hence $t_{m} e_{0} \in C$; but $e=\left(t_{m} e_{0}\right) e=t_{m}\left(e_{0} e\right)=t_{m} e$, a contradiction, since $t_{m} \in S \backslash C$.

Lemma 5. If $x \in S \backslash C$ and $c \in C$, then $x c, c x \in S \backslash C$ and $x T \cap C=\varnothing$.

Proof. $e x=x e \neq e$ and $c e=e c=e$, so $(x c) e=x(c e)=x e \neq e$ and $(c x) e=e(c x)=e x \neq e$.

Lemma 6. Bd $S \cap C$ is an arc from a to $a^{-1}$ for some $a \in C$.

Proof. Let $a \in \mathrm{Bd} S \cap C$ such that $a \neq 1$. Since $C$ is acyclic, $\mathrm{Bd} S \not C$, and since inversion is a homeomorphism on $\mathrm{Bd} S$, it will suffice to show that one of the arcs of $\mathrm{Bd} S$ from $a$ to 1 is contained in $C$. Let $A$ and $B$ be these arcs. Suppose there exist $x \in A \backslash C$ and $y \in B \backslash C$. Then the connected set $T_{1} \cup a T_{1} \subset C$ separates either $x$ or $y$ from 0 , while $x T \cup y T$ is a connected subset of $S \backslash C$ containing $x, y$, and 0 , a contradiction.

Lemma 7. $\operatorname{Bd}(S \backslash C) \cap C=a T_{1} \cup a^{-1} T_{1}$.

Proof. Clearly $a T_{1} \cup a^{-1} T_{1} \subset \operatorname{Bd}(S \backslash C) \cap C$, and since $1 \notin a T_{1} \cup a^{-1} T_{1}$, this set separates $S$ and the components separated from 0 are contained 
in $C$. Let $\left\{t_{n}\right\}$ be a sequence in $S \backslash C$ converging to $a$ such that $t_{n} e \neq t_{n}^{-1} e$, and let $H_{n}$ be the arc of $H(e)$ from $t_{n} e$ to $t_{n}^{-1} e$ containing $e$. Now consider the arcs $C_{n}=t_{n} T_{1} \cup H_{n} \cup t_{n}^{-1} T_{1}$, noting $H_{n} \cap t_{n} T_{1}=\left\{t_{n} e\right\}, H_{n} \cap t_{n}^{-1} T_{1}=$ $\left\{t_{n}^{-1} e\right\}$, and $t_{n} T_{1} \cap t_{n}^{-1} T_{1}=\varnothing$. The arcs $C_{n}$ separate $C$ from 0 , and clearly $\left\{C_{n}\right\}$ converges to $a T_{1} \cup a^{-1} T_{1}$. Now if $x \in S$ is such that $x$ and 0 are not separated by $a T_{1} \cup a^{-1} T_{1}$, then there is an $m$ such that $C_{m}$ separates $x$ from $C$. Hence $x \notin C$ and $a T_{1} \cup a^{-1} T_{1}=\operatorname{Bd}(S \backslash C) \cap C$.

THEOREM 1. S is the min cone over the circle group and $e=1$.

Proof. By Lemma 6, the Bd $S \cap C$ is an arc with end points $a$ and $a^{-1}$. It follows from Lemmas 5 and 7 that $a a^{-1} \in a T_{1} \cup a^{-1} T_{1}$. Therefore either $a a^{-1}=a f$ for some $f \in T_{1}$ and $a^{-1}=a^{-1} a a^{-1}=a^{-1} a f \in E$, a contradiction, or $a a^{-1}=a^{-1} f$ and $a=a a^{-1} a=a^{-1} f a \in E$, again a contradiction.

The referee observed that if $S$ is a Clifford semigroup with identity on the 2-cell, whose set $E$ of idempotents has a cut point, then Lemmas 1 and 2 follow with slight modifications, where $e$ can again be considered the maximum idempotent of $T$ for which $e S e$ is a min cone over the circle group. Continuing the referee's suggestions, let $T_{1}=\{t \in T: e \leqq t\}$. Then $\theta: T_{1} \times S \rightarrow S$ defined by $\theta(t, x)=t x$ is a homotopy retract of $S$ onto $e S e$ and by the homology properties of the circle and disk, this implies $e \cdot \operatorname{Bd} S=H(e)$. Now if $g \in \mathrm{Bd} S$ is such that $e g$ has infinite order, then multiplication by $e$ maps the closed subgroup generated by $g$ homomorphically onto $H(e)$, and since dimension cannot be raised by a homomorphism, $g \in H(e)$. Let $C=\{s \in S: s e=e\}$; again $\mathrm{Bd} S \cap C$ is a connected arc containing 1 with end points $a$ and $b$. But there exist sequences of elements of infinite order in $H(e) \cap \mathrm{Bd} S$ converging to $a$ and $b$. Hence $a=e=b$ and $e=1$.

The following more general theorem now follows, although in the case of Clifford semigroups one must take into consideration the fact that idempotents need not commute.

THEOREM 2. If $S$ is an inverse semigroup or Clifford semigroup with identity on the 2-cell whose set of idempotents has a cut point, then $S$ is the min cone over the circle group.

The author has some results in the case when the set of idempotents has no cut point and in a later paper this case will be considered, with a view to completing a characterization of inverse and Clifford semigroups with identity on the 2-cell.

The author wishes to express his appreciation to Professor R. J. Koch for his encouragement and helpfulness during the preparation of this paper. 


\section{REFERENCES}

1. L. W. Anderson and R. P. Hunter, The $\mathscr{H}$-equivalence in compact semigroups, Bull. Soc. Math. Belg. 14 (1962), 274-296. MR 27 \#1920.

2. A. H. Clifford and G. B. Preston, The algebraic theory of semigroups. Vols. I, II, Math. Surveys, no. 7, Amer. Math. Soc., Providence, R.I., 1961, 1967. MR 24 \#A2627; 36 \#1558.

3. K. H. Hofmann and P. S. Mostert, Elements of compact semigroups, Charles E. Merrill, Columbus, Ohio, 1966. MR 35 \#285.

4. W. Hurewicz and H. Wallman, Dimension theory, Princeton Math. Series, vol. 4, Princeton Univ. Press, Princeton, N.J., 1941. MR 3, 312.

5. R. J. Koch and A. D. Wallace, Notes on inverse semigroups, Rev. Roumaine Math. Pures Appl. 9 (1964), 19-24. MR 31 \#1662.

6. R. J. Koch, Arcs in partially ordered spaces, Pacific J. Math. 9 (1959), 723-728. MR 21 \#7269.

7. A. D. Wallace, Cohomology, dimension and mobs, Summa Brasil. Math. 3 (1953), 43-55. MR 15, 336.

Department of Mathematics, West Chester State College, West Chester, Pennsylvania 19380

Department of Mathematics, Northern Illinois University, DeKalb, Illinois 60115 